\title{
The Peer Reader Program: Supporting the Regional English Language Education Research Community
}

\author{
Kelly Kimura \\ Soka University, Tokyo, Japan
}

\begin{abstract}
Language Education in Asia (LEiA) exists to offer regional authors a place to participate in the English language education research community and share their research, teaching practices, and commentaries. However, each year, the LEiA Editorial Board regretfully has to decline a number of papers on topics that would be of interest to our readers. Many times, bringing these papers to publication would require more time and resources than are available throughout our publication process.
\end{abstract}

Until this year, we at LEiA could only hope that these authors would be able to find publication opportunities elsewhere. At the same time, we were concerned that some authors, particularly those writing in their second or third language, would be discouraged about continuing to seek publication for papers that had been rejected once. John Middlecamp and I wondered what support these authors had. While LEiA has encouraged authors to seek colleagues to read and critique submissions, it was possible that not all authors would have local access to extensive, constructive feedback on academic writing for publication in English, whether in developing or developed contexts in Asia. This kind of feedback might be necessary to help these authors succeed in strengthening their papers and achieving publication.

This year, to address this need, John Middlecamp and Alan Klein created an initiative called the Peer Reader Program, with the larger goal of supporting the development of the English language education research community in Asia, particularly in the ASEAN region. Separate from the LEiA publication process, the program provides a free, short-term service to nonnative English-speaking authors whose papers were submitted to LEiA but not chosen to undergo review.

The feedback in the program is provided by Peer Readers, volunteers who have had papers published in peer-reviewed journals. The Peer Reader positions offer the volunteers an opportunity to further participate in the academy by using their knowledge and publication experience to support other authors. The first group of Peer Readers was recruited from the ranks of authors who have published with LEiA. The strong positive response was a pleasant surprise: $19 \mathrm{LEiA}$ authors working or studying in 10 countries volunteered. This was the first opportunity for some of the Peer Readers to formally provide professional guidance to a peer author. Regardless of experience, the feedback offered has been thorough and thoughtful, and we look forward to the continued participation of the Peer Readers.

The process is kept simple and relatively short. Papers declined by the LEiA Editorial Board at the screening stage are sent to John and Alan, the Peer Reader Coordinators, for evaluation of

Language Education in Asia, 2016, 71), 1-3. http://dx.doi.org/10.5746/LEiA/16/V7/11/A01/Kimura 
their suitability for the program. Papers are selected for the relevancy of their research topics to the ASEAN context. Authors of these papers are invited to participate in the program. If they accept, the coordinators send the authors' blinded papers to the Peer Readers. The Peer Readers, who are anonymous to the authors, examine the papers using a feedback form. They send their feedback to the coordinators, who in turn send it to the authors. The authors then have a limited period to ask questions about the feedback or ask for comments on a revision based on the feedback. The entire process may take up to approximately two months.

This year, thanks to the large number of Peer Reader volunteers, the program was piloted with authors of seven papers, more than twice the number first envisaged. While the long-term results of the program - publication of the papers - will not be apparent for some time, the pilot was successful in providing the participating authors with valuable peer and community support. Now it is up to these authors to take the initiative to revise and submit their papers to a publication. As the authors know, participation in the program does not guarantee eventual publication in LEiA, and they can freely choose where to submit their revised papers. Because the perspectives of each Peer Reader and future reviewer may differ, the authors can expect that pursuing publication will involve further revisions. We wish them the best in having their work published in LEiA or another journal.

I would like to thank John Middlecamp and Alan Klein for all of their work on the Peer Reader Program, and the inaugural group of volunteer Peer Readers, listed here, for their generosity in providing feedback to the participating authors.

Dr Scott Aubrey, Kansai University, Japan

Mr Brendan Bartlett, Australian Catholic University, Australia

Ms Bui Le Diem Trang, Victoria University of Wellington (PhD Student), New Zealand

Mr Peter J. Collins, Tokai University Shonan Campus, Japan

Ms Doan Linh Chi, Khan Hoa University, Vietnam

Dr Krystyna U. Golkowska, Weill Cornell Medicine - Qatar, Qatar

Mr Steven Graham, American University of Phnom Penh, Cambodia

Mr Michael Guest, University of Miyazaki, Japan

Dr Susan Gwee, English Language Institute of Singapore, Singapore

Ms Monica Hamciuc, Miyazaki International College, Japan

Dr Linda M. Hanington, National Institute of Education, Nanyang Technological University,

Singapore

Ms Nina Inayati, University of Muhammadiyah Malang, Indonesia

Mr Zabih O. Javanbakht, Department of Education, Iran

Mr Kea Leaph, Secretariat-General of the National Assembly of the Kingdom of Cambodia,

Cambodia

Mr Le Duc Manh, Haiphong University, Vietnam

Ms Channy Meng, Phnom Penh International University, Cambodia

Dr Nguyen Thi Thuy Loan, Kalasin University, Thailand

Ms Huong Quynh Tran, Monash University, Australia

Mr Michael Wilkins, Kwansei Gakuin University, Japan

Turning to the current volume, this is the first year that LEiA has one submission date for papers for both issues. This issue, Volume 7, Issue 1, has three research papers and one teaching practice paper.

In the first research paper, Viet Thi Nguyen investigates differences in language learning strategy use between English majors and non-English majors at a university in Vietnam. Next, Leander S. Hughes examines the effects of parsed and block text formats in online extensive reading in English on the reading proficiency of Japanese university students. In the last paper in the research category, Thi Khanh Doan Nguyen, Steven Pickford, and Barbra McKenzie look at why learner resistance occurs in the language learning classroom in Vietnam, using the activity theory as a framework, and how resistant learners are viewed by their teachers. 
The teaching practice paper in this issue is by Rosyi Arina and Nur Kamilah in Indonesia. The authors explain how students read local folktales in English for extensive reading and then make and present posters about the folktales, encouraging both extensive reading in English and learning about their culture.

The publication of these four papers for Issue 1 reveals just a glimpse of the dedicated work that the members of the editorial team put into screening all submissions (much gratitude here to John Middlecamp), advising authors through revisions, copy editing, and formatting. The reviewers on the Editorial Board generously supported authors with detailed feedback to guide their revisions. We also appreciate the continued support of the publication by the Advisory Board and IDP Education (Cambodia).

Thank you to all authors who submitted papers, and congratulations to the authors whose papers are published in this issue.

Finally, we hope our readers will find the papers in this issue of interest. 